\title{
Modeling of the Non-Isothermal Crystallization Kinetics of Polyamide 6 Composites During Thermoforming
}

\author{
Daniel Kugele ${ }^{1, a)}$, Dominik Dörr ${ }^{1, b)}$, Florian Wittemann ${ }^{1}$, Benjamin Hangs ${ }^{2}$, Julius \\ Rausch $^{3}$, Luise Kärger ${ }^{1}$ and Frank Henning ${ }^{1,2}$ \\ ${ }^{1}$ Karlsruhe Institute of Technology (KIT), Institute of Vehicle System Technology, Chair of Lightweight Technology, \\ Rintheimer-Querallee 2, 76131 Karlsruhe, Germany \\ ${ }^{2}$ Fraunhofer Institute for Chemical Technology, Polymer Engineering Department, Joseph-von-Fraunhofer-Str. 7, \\ 76327 Pfinztal, Germany \\ ${ }^{3}$ AUDI AG, Technology Development Fiber-Reinforced Polymers, 74148 Neckarsulm, Germany

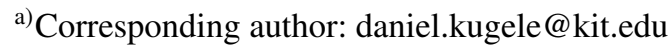 \\ b) dominik.doerr2@kit.edu
}

\begin{abstract}
The combination of thermoforming processes of continuous-fiber reinforced thermoplastics and injection molding offers a high potential for cost-effective use in automobile mass production. During manufacturing, the thermoplastic laminates are initially heated up to a temperature above the melting point. This is followed by continuous cooling of the material during the forming process, which leads to crystallization under non-isothermal conditions. To account for phase change effects in thermoforming simulation, an accurate modeling of the crystallization kinetics is required. In this context, it is important to consider the wide range of cooling rates, which are observed during processing. Consequently, this paper deals with the experimental investigation of the crystallization at cooling rates varying from $0.16 \mathrm{~K} / \mathrm{s}$ to $100 \mathrm{~K} / \mathrm{s}$ using standard differential scanning calorimetry (DSC) and fast scanning calorimetry (Flash DSC). Two different modeling approaches (Nakamura model, modified Nakamura-Ziabicki model) for predicting crystallization kinetics are parameterized according to DSC measurements. It turns out that only the modified Nakamura-Ziabicki model is capable of predicting crystallization kinetics for all investigated cooling rates. Finally, the modified Nakamura-Ziabicki model is validated by cooling experiments using PA6-CF laminates with embedded temperature sensors. It is shown that the modified Nakamura-Ziabicki model predicts crystallization at non-isothermal conditions and varying cooling rates with a good accuracy. Thus, the study contributes to a deeper understanding of the non-isothermal crystallization and presents an overall method for modeling crystallization under process conditions.
\end{abstract}

\section{INTRODUCTION}

The combination of thermoforming processes of continuous-fiber reinforced thermoplastics and injection molding (overmolding process) offers a high potential for cost-effective use in automobile mass production. The advantageous short cycle time, the additional possibility of designing lightweight, complex and functionalized components are one of the major benefits of such hybrid material systems. The thermal behavior of the laminate along the entire process chain, consisting of heating, transfer, thermoforming and overmolding is crucial for manufacturing [1-3]. At this background, especially the crystallization kinetics of semi-crystalline polymers have a significant influence on the forming process. Since the viscosity increases rapidly during the transition from the molten state into the solid state [4], the crystallization defines a crucial process limit for defect-free forming.

Both, the experimental investigations, as well as the modeling of crystallization kinetics have to consider the wide range of cooling rates observed during the process. Commonly, the crystallization is studied using differential scanning calorimetry (DSC). However, cooling rate is limited to a maximum of approximately $2.5 \mathrm{~K} / \mathrm{s}$ or in special cases $10 \mathrm{~K} / \mathrm{s}$ for standard DSC [5]. In order to investigate higher cooling rates, a break-through was the development of extremely fast-operating calorimeters, which are presented by Schick et al. [6, 7], Allen et al. [8] and Mathot et al. [9]. Based on this method, the experimental investigation of the crystallization at high cooling rates $(>10 \mathrm{~K} / \mathrm{s})$ is possible. This allows the parametrization and validation of crystallization models at process relevant cooling rates. 
In this work, initially the applied approaches for modeling of crystallization kinetics are outlined shortly. Subsequently, experimental results of DSC and Flash DSC measurements for a PA6-CF UD-tape are presented. These experimental results are adopted for parameter identification of the investigated crystallization modeling approaches. Finally, crystallization modeling is validated by applying a parameterized model to experimental tests on component level.

\section{CONSTITUTIVE MODELING}

\section{Modeling of the non-isothermal crystallization kinetics}

A large number of earlier investigations dealt with models to analyze the non-isothermal crystallization kinetics. Comprehensive reviews are provided by Di Lorenzo et al. [10], Mandelkern [11], Long et al.[12] and Ding et al. [13]. Almost all of the presented models are based on the work of Avrami [14], where the general form of the Avrami equation for isothermal conditions is given by

$$
X(t)=1-\exp \left(-k t^{n}\right) .
$$

$X(t)$ is the crystallinity fraction at the time $t, k$ the crystallization rate constant and $n$ the so-called Avrami index. Both material constants $(k, n)$ depend on the given morphology and type of nucleation.

To study the non-isothermal crystallization, several models have been developed. Ozawa [15] modified Avrami's equation to describe non-isothermal crystallization. This theory works well for a variety of materials and applications. Nevertheless, Patel [16] listed several drawbacks and concludes that the Ozawa theory is not suitable for non-isothermal crystallization within a wide range of cooling rates. Despite this, Nakamura [17-19] introduced another extension of the Avrami equation, where the degree of phase transformation is given by

$$
\left.X(t)=1-\exp \left[-\left(\int_{0}^{t} K(T(\tau)) d \tau\right)\right)^{n}\right] \text { with } \quad K(T)=k(T)^{1 / n} .
$$

Instead of modeling the crystallization rate constant $K(T)$ by an analytic function, Guo and Isayec [20] implemented an expression of Hoffmann and Lauritzen [21] to describe the temperature dependent crystallization rate constant by

$$
K(T)=(\ln 2)^{1 / n}\left(\frac{1}{t_{1 / 2}}\right) \exp \left(-\frac{U^{*}}{R\left(T-T_{\infty}\right)}\right) \exp \left(-\frac{K_{k}}{T \Delta T f}\right) .
$$

This theory was also applied by Hoffmann [22] and Patel [16]. Furthermore, Ziabicki [23, 24] extended the Avrami equation to predict the non-isothermal crystallization kinetics by an empirical relation for the crystllization rate constant by

$$
K(T)=K_{\max } \exp \left[\frac{-4 \ln 2\left(T-T_{\max }\right)^{2}}{D^{2}}\right] .
$$

In this theory, the crystallization rate constant in relation to temperature is represented by a Gaussian function depending on the parameters $K_{\max }, D$ and $T_{\max }$. Following this approach, Hoffmann [22] and Sierra [25] presented a modified Nakamura-Ziabicki model, which is based on the combination of Nakamura's approach (Equation 2) and Ziabicki's approach for the description of $K(T)$ (Equation 4).

\section{Heat transfer modeling}

Heat transfer modeling is applied to predict the temperature distribution in the test specimens. Based on Fourier's law, the three-dimensional transient heat conduction problem is given by

$$
\frac{\partial T}{\partial t}=\frac{1}{\rho c_{p}}\left[\left(k_{x} \frac{\partial^{2} T}{\partial x^{2}}\right)+\left(k_{y} \frac{\partial^{2} T}{\partial y^{2}}\right)+\left(k_{z} \frac{\partial^{2} T}{\partial z^{2}}\right)\right]+\frac{\dot{q}_{s}}{\rho c_{p}} \quad \text { with } \quad \dot{q}_{s}=\rho_{m} \Delta H_{f} X_{\infty} \frac{d X}{d t}\left(1-V_{f}\right),
$$

where $k_{i}$ are the material constants for the orthotropic thermal conductivity, $\rho$ the density and $c_{p}$ the specific heat. The heat flow caused by the phase change of the polymer $\left(\dot{q}_{s}\right)$ considers the density of the polymer $\left(\rho_{m}\right)$, the enthalpy of crystallization $\left(\Delta H_{f}\right)$, the maximum fraction of transformation (degree of crystallization) $\left(X_{\infty}\right)$ and the fiber volume $\left(V_{f}\right)$. 
For this study, heat transfer modeling is reduced to thickness direction, where the governing one-dimensional heat transfer equation for a finite region $0 \leq z \leq d$ is given by

$$
\frac{d T}{d t} \approx \frac{\partial T}{\partial t}=\frac{1}{\rho c_{p}}\left(k_{z} \frac{\partial^{2} T}{\partial z^{2}}\right)+\frac{1}{\rho c_{p}}\left(\rho_{m} \Delta H_{f} X_{\infty} \frac{d X}{d t}\left(1-V_{f}\right)\right)
$$

This heat equation is solved by a Finite Difference Method under consideration of the boundary conditions convection and heat conduction.

\section{PARAMETRIZATION AND VALIDATION}

\section{Differential scanning calorimetry}

In the scope of this study, unidirectional carbon fiber reinforced tape with a PA6 matrix (BASF Ultratape ${ }^{\mathrm{TM}}$ ) and a fiber content by weight of $60 \%$ and a density of $1.46 \mathrm{~g} \mathrm{~cm}^{-3}$ is investigated. Differential scanning calorimetry (DSC) measurements were conducted with a Perkin-Elmer DSC 8000 in aluminium pans and nitrogen atmosphere according to DIN EN ISO 11357-1. The material was dried under vacuum at $80^{\circ} \mathrm{C}$ for $10 \mathrm{~h}$ prior to experimentation. The samples with a weight of approximately $10 \mathrm{mg}$ were heated with a rate of $0.167 \mathrm{~K} / \mathrm{s}$ starting at $30^{\circ} \mathrm{C}$. The samples were hold at $240{ }^{\circ} \mathrm{C}$ for $2 \mathrm{~min}$ and then cooled with cooling rates of $0.16 \mathrm{~K} / \mathrm{s}, 0.33 \mathrm{~K} / \mathrm{s}, 0.5 \mathrm{~K} / \mathrm{s}, 0.66 \mathrm{~K} / \mathrm{s}$ and $1 \mathrm{~K} / \mathrm{s}$. Fast differential scanning calorimetry with a Mettler-Toledo Flash DSC 1 was performed at the Institute of Physics at the University of Rostock. Small samples were cut out of the composite material, heated with $100 \mathrm{~K} / \mathrm{s}$ up to $280^{\circ} \mathrm{C}$, held at this temperature for $3 \mathrm{~s}$ and then cooled with cooling rates of $5 \mathrm{~K} / \mathrm{s}, 15 \mathrm{~K} / \mathrm{s}, 30 \mathrm{~K} / \mathrm{s}, 50 \mathrm{~K} / \mathrm{s}$ and $100 \mathrm{~K} / \mathrm{s}$. The results of the standard DSC measurements (cooling rates between $0.16 \mathrm{~K} / \mathrm{s}$ and $1 \mathrm{~K} / \mathrm{s}$ ) are shown in Figure $1 \mathrm{a}$ ). The typical shift of the crystallization peak to lower temperatures with increasing cooling rate is observed. The Flash DSC results (Figure $1 \mathrm{~b}$ )) show the same characteristic material behavior.

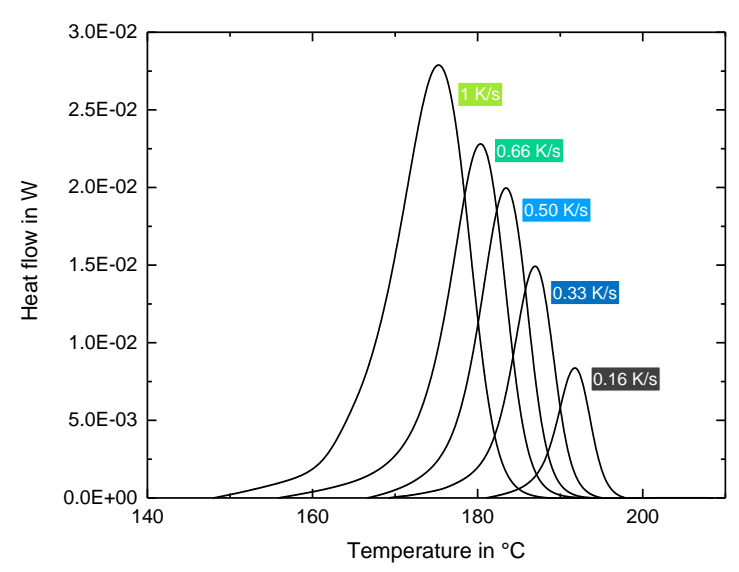

(a)

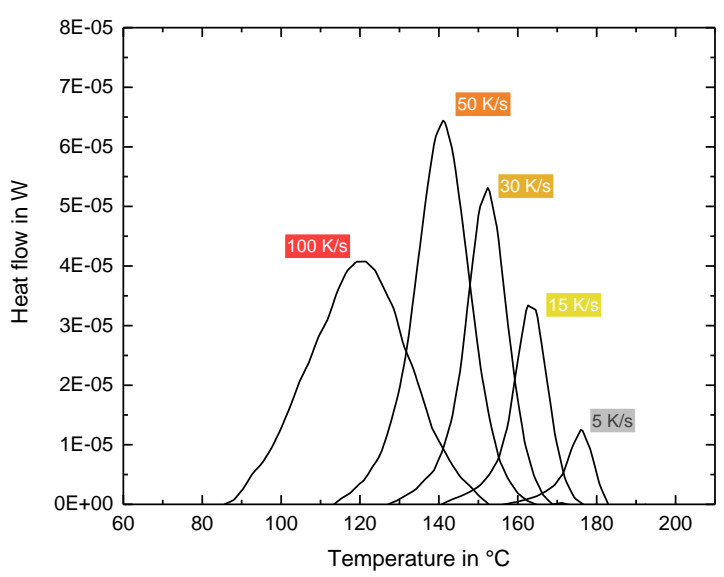

(b)

FIGURE 1. Data for non-isothermal crystallization at different cooling rates derived from standard DSC measurements (a) and Flash DSC measurements (b).

\section{Parametrization of the crystallization kinetic models}

Two different approaches for modeling crystallization kinetics are investigated in the following. Namely, these are Nakamura's model (Equation 2 and 3) and the so-called modified Nakamura-Ziabicki model (Equation 2 and 4). Parameters are identified using a genetic optimization algorithm based on the relative cristallinity determined by the DSC measurements presented above.

In the following, relative crystallinity is adopted, which is derived from the experimental data shown in Figure 1. For the relative crystallinity, the entire spectrum of the non-isothermal crystallization can be merged into one diagram (cf. e.g. Figure 2 a)). The evolution of relative crystallinity starts at 0 , where the polymer is in its molten state and no 
crystalline structures exist. With decreasing temperature, the crystalline structures grow until a maximum value of 1 is reached. These characteristic curves are used in the following for the parametrization of the investigated approaches for modeling crystallization kinetics.

Nakamura's model: Initially, the above described procedure for parameter identification is applied only for the standard DSC data and hence for cooling rates lower than $1 \mathrm{~K} / \mathrm{s}$ (cf. Figure $2 \mathrm{a}$ ). It can be seen, that the Nakamura model predicts the non-isothermal crystallization with a good accuracy for low cooling rates, which are considered for parameter identification. However, extrapolation for higher cooling rates shows a poor accuracy.

Furthermore, the entire spectrum of cooling rates is considered for parameter identification for Nakamura's model (cf Figure 2 b). Overall, a moderate but not sufficient prediction accuracy is observed. Especially the prediction quality for the onset of crystallization, which is crucial for thermoforming, is poor.

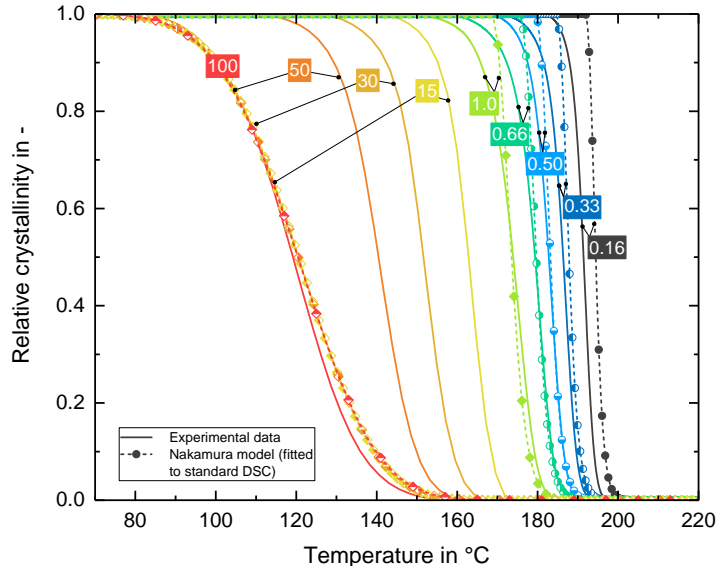

(a)

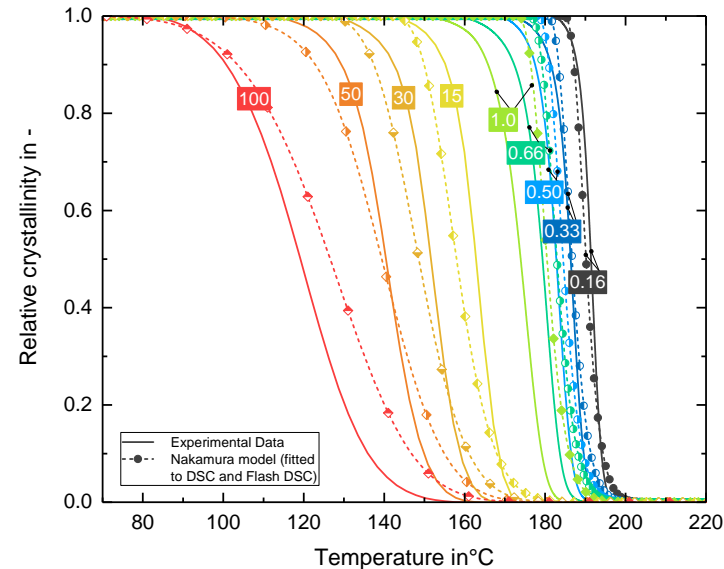

(b)

FIGURE 2. Relative crystallinity at different cooling rates derived from standard DSC and Flash DSC measurements. Comparison of experimental data and prediction of Nakamura model fitted to standard DSC data (a). Prediction accuracy of Nakamura model fitted to standard DSC and Flash DSC data (b).

Modified Nakamura-Ziabicki model: Besides Nakamura's model, also the the so-called modified Nakamura-Ziabicki model (Equation 2 and 4) is investigated (cf. Figure 3). For this approach, the parameters $K_{\max }$, $D$ and $T_{\max }$ are identified for each cooling rate. The parameter sets for each cooling rate are subsequently applied to regression functions, to continuously interpolate the model parameters within the investigated range of cooling rates. It turns out, that this approach is capable of describing crystallization kinetics of each of the investigated cooling rates.

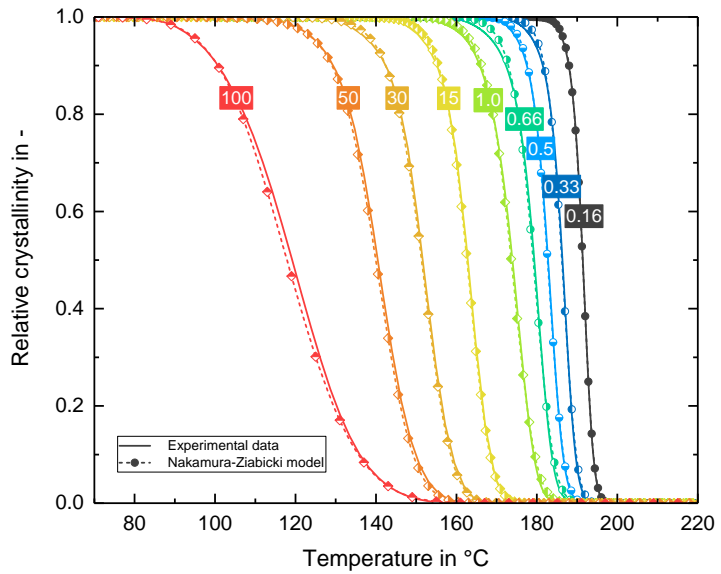

FIGURE 3. Comparison of experimental data and prediction of the modified Nakamura-Ziabicki model. 


\section{Validation under process conditions}

In the following, the modified Nakamura-Ziabicki model is adopted for validation on component level, as only this model has proven to be capable of describing crystallization kinetics for all of the consdiered cooling rates. Validation is carried out by cooling experiments with laminates and embedded temperature sensors.

Laminate transfer: Cooling behavior during a transfer process (forced convection with air velocity of $3 \mathrm{~m} / \mathrm{s}$ ) is performed with the experimental setup presented by Kugele et al. [26]. The temperature measurement in the midplane of the laminate is plotted in Figure 4 a) (black line). It can be seen, that the temperature gradient has a characteristic peak during crystallization. This information is used to indicate the crystallization rate and to compare the simulation to the measurement. The similarity of experiment and simulation proves that the modified NakamuraZiabicki model is capable of predicting the onset of crystallization.

Tool contact: The same procedure is repeated for experiments with higher cooling rates. For this purpose, the experimental setup presented by Kugele et al. [27] is used to investigate crystallization during cooling experiments of laminates in a temperature-controlled press mold. As indicated in Figure 4 b), the simulation predicts the start of the crystallization again with a great accordance.

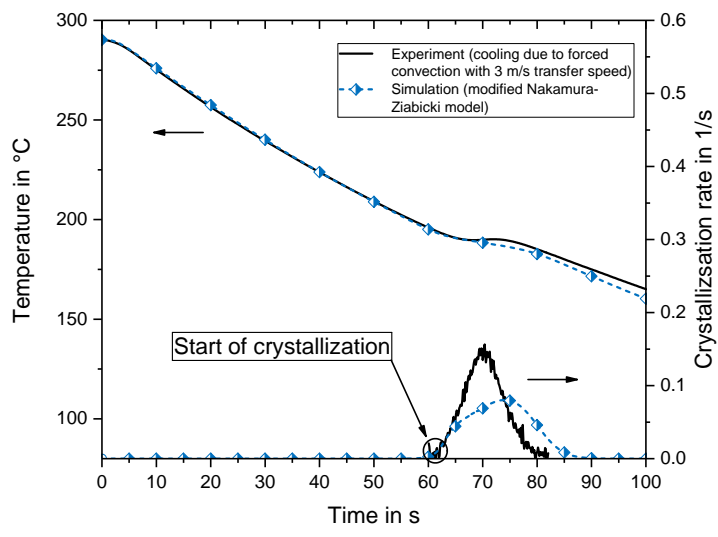

(a)

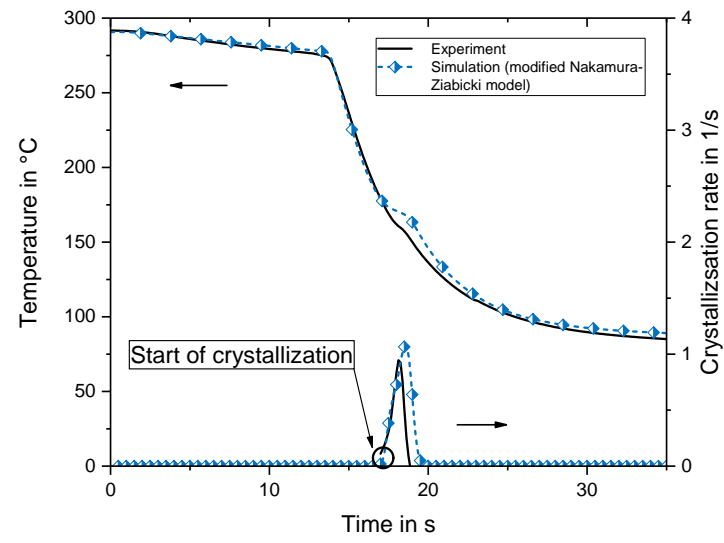

(b)

FIGURE 4. Validation of modified Nakamura-Ziabicki model by comparing the simulated and measured temperature curves and crystallization rates within the mid-plane of laminates at two different cooling conditions: cooling due to forced convection (a); cooling as a consequence of heat transfer between laminate and steel mold (b).

\section{CONCLUSION}

In the present study, the non-isothermal crystallization kinetics of PA6-CF is investigated. For this purpose, DSC and Flash DSC measurements are presented and discussed. Furthermore, Nakamura's model, as well as the modified Nakamura-Ziabicki model is parameterized according to the experimental data. It is shown that both models can be used to simulate the non-isothermal crystallization kinetics. However, only the modified Nakamura-Ziabicki is capable of predicting crystallization kinetics for the wide range of considered cooling rates. Beyond that, it is shown that an extrapolation for modeling crystiallization kinetics for high cooling rates, based on a parametrization according to standard DSC measurements, leads to a significant error for the prediction of crystallization kinetics. This makes Flash DSC measurements crucial for the characterization of crystillization kinetics in thermoforming processes.

Finally, the modified Nakamura-Ziabicki model is validated by cooling experiments using PA6-CF laminates with embedded temperature sensors. It is successfully demonstrated that the model predicts the onset of crystallization at non-isothermal conditions and varying cooling rates with a good accuracy. Thus, the study contributes to a deeper understanding of the non-isothermal crystallization and presents an overall method for modeling crystallization under 
process conditions. This can be used to improve the prediction accuracy of forming simulation as well as to optimize the process window considering the non-isothermal crystallization.

\section{ACKNOWLEDGMENTS}

The authors thank Prof. Schick and A. Wurm (University of Rostock, Institute of Physics) for the support and the opportunity to perform the fast scanning calorimetry in Rostock. The support of BASF Ludwigshafen for providing DSC data is also gratefully acknowledged. Furthermore, the authors would like to thank the German Federal Ministry of Education and Research for the funding of the project SMiLE (03X3041P), for which parts of the DSC measurements are carried out.

\section{REFERENCES}

[1] J. Rausch and D. Kugele, Temperature Monitoring of Thermoplastic Laminates in an Automated Process Chain - Potential for Enhanced Mechanical Properties and Effective Processing: SAMPE Europe, 2016.

[2] E. Guzman-Maldonado, N. Hamila, N. Naouar, G. Moulin, and P. Boisse, Materials \& Design 93, 431-442 (2016).

[3] H. Lessard, G. Lebrun, A. Benkaddour, and X. Pham, Composites Part A: Applied Science and Manufacturing 70, 59-68 (2015).

[4] Christian Brauner, Christian Peters, Franziska Brandwein, Axel Herrmann, Journal of Composite Materials (2013).

[5] PerkinElmer, Technical Specifications for the DSC 8000/8500 Differential Scanning Calorimeter, .

[6] C. Schick, Analytical and bioanalytical chemistry 395, 1589-1611 (2009).

[7] E. Zhuravlev and C. Schick, Thermochimica Acta 1-13 (2010).

[8] M. Y. Efremov, E. A. Olson, M. Zhang, F. Schiettekatte, Z. Zhang, and L. H. Allen, Review of Scientific Instruments 75, p. 179 (2004).

[9] V. Mathot, M. Pyda, T. Pijpers, G. Vanden Poel, E. van de Kerkhof, S. van Herwaarden, F. van Herwaarden, and A. Leenaers, Thermochimica Acta 522, 36-45 (2011).

[10] M. L. Di Lorenzo and C. Silvestre, Progress in Polymer Science 24, 917-950 (1999).

[11] L. MANDELKERN, Crystallization of Polymers: Kinetics and mechanisms, Second Edition (Cambridge University Press, Cambridge, 2004).

[12] Long, (1995).

[13] Z. Ding and J. E. Spruiell, Journal of Polymer Science Part B: Polymer Physics 35, 1077-1093 (1997).

[14] M. Avrami, The Journal of Chemical Physics 7, p. 1103 (1939).

[15] T. Ozawa, Polymer 150-158 (1970).

[16] R. M. Patel and J. E. Spruiell, Polymer Engineering \& Science 730-738 (1991).

[17] Nakamura, Journal of Applied Polymer Science 1077-1091 (1972).

[18] Nakamura, (1973).

[19] Nakamura, (1974).

[20] X. Guo, A. I. Isayev, and L. Guo, Polymer Engineering \& Science 1999, p. 2096 (1999).

[21] J. D. Hoffman, G. T. Davis, and J. I. Lauritzen, Treatise on Solid State Chemistry 497-614 (1976).

[22] S. Hoffmann, (2003).

[23] A. Ziabicki, Fundamentals of Fibre Formation: The Science of Fibre Spinning and Drawing (John Wiley \& Sons Ltd (1. Januar 1976), 1976).

[24] A. Ziabicki, Colloid \& Polymer Science 274, 209-217 (1996).

[25] J. D. Sierra, M. d. P. Noriega, and J. F. Gomez, Zeitschrift Kunststofftechnik 2006 (2006).

[26] D. Kugele, J. Rausch, J. Kriegseis, K. Gündisch, L. Kärger, and F. Henning, On the Thermal Behavior of Thermoplastic Laminates During Transfer - A Novel Wind-Tunnel Approach: 17th European Conference on Composite Materials, 2016.

[27] D. Kugele, J. Rausch, P. Müller, L. Kärger, and F. Henning, Temperature Distribution in Thickness Direction of Thermoplastic Laminates During Thermoforming: International Conference on Automotive Composites, 2016. 